\title{
संगीत और काव्य साहित्य में अन्तर्सम्बन्ध
}

\section{Shivam Mishra}

Research Scholar, Instrumental Department, Faculty of Performing Arts, Banaras Hindu University, Varanasi (U.P.)

सार संक्षेपिका

भारत के सांस्कृतिक जीवन में काव्य और संगीत का विशेष स्थान है। यदि उपासना भारतीय जीवन शेली का अभिन्न अंग है, तो काव्य और संगीत उपासना के अभिन्न अंग हैं। इसका अर्थ यह नहीं की उपासना से इतर जीवन में भारतीय कलाकारों ने कला को ग्रहण नहीं किया। वेदमंत्रों में उदात्त, अनुदात्त, स्वरित रूप से भारत के प्राचीनतम साहित्य में संगीत के स्वर जगते हैं। यही नहीं श्रीकृष्ण कथित-"वेदानांसामवेदोस्मि" के उद्घोष में भी सामवेद का सांगीतिक महत्व प्रखर हुआ है तथा बाद में भी आदिकाव्य रामायण से पुराण, संस्कृत और पाली साहित्य तक काव्य संगीत उपासना का ही आंचल पकड कर सामने आये।

बीज शब्द

संगीत, काव्य साहित्य

\section{ABSTRACT}

Poetry and music have a special place in the cultural life of India. If worship is an integral part of the Indian way of life, poetry and music are integral parts of worship. This does not mean that Indian artists did not accept art in life other than worship. In the Vedamantras, the sublime, transcendent, vocal forms of music in India's oldest literature. Not only this, the musical significance of Samaveda has also become evident in the announcement of Sri Krishna's alleged - "Vedansamamvedosmi" and later also came to hold the flame of poetic music worship from Adikavya Ramayana to Purana, Sanskrit and Pali literature.

Keywords

Sangeet, Poem, Litrature

भूमिका

“संगीत" शब्द की उत्पत्ति, "गीत" शब्द में "सम" उपसर्ग लगा कर बना है। "सम" मतलब सहित और "गीत" यानी "गान"। गान के साथ अर्थात् अंग भूत क्रियाओं (नृत्य) व वादन के सहित किया हुआ कार्य संगीत है। संगीत वह ललित कला है जिसके अंतर्गत स्वर और लय के द्वारा हम अपने भावों को प्रकट करते हैं।

साहित्य और संगीत मनुष्य के भावों को व्यक्त करने के महत्वपूर्ण माध्यम है। साहित्य और संगीत के समन्वय से अलौकिक सौन्दर्य की सृष्टि होती है, जो मानव मन को सच्चिदानंद की अनुभूति प्रदान करता है। वाराहोपनिषद् में वर्णित है कि-संगीत सम्यक् गीत है, भागवत महापुराण में नृत्य तथा वाद्य यंत्रों के साथ प्रस्तुत गायन को संगीत बतलाया गया है। संगीत का मुख्य उद्देश्य मनोरंजन करने वाला माना जाता है, यही उद्देश्य साहित्य का भी होता है। संगीत नाद बह्म और ताल बह्म का साक्षात्कार कराता है और साहित्य अक्षर ब्रह्म और शब्द ब्रह्म का। दोनों ही कलाओं का अत्यंत घनिष्ठतम सम्बन्ध है। इसका प्रत्यक्ष उदाहरण माँ सरस्वती हैं, जिनके एक हाथ में वीणा और दूसरे में पुस्तक है। भर्तृहरिकृत नीतिसतकम् में कहा गया है-“"साहित्य संगीत कला विहीन: साक्षात् पशु पुच्छ विषाण हीन:"। अर्थात् साहित्य, संगीत और कला से विहीन मनुष्य साक्षात् पशु के समान है। 
वाक्यार्थ साहित्य और संगीत के सह अस्तित्व को पूर्णरूपेण स्पष्ट करता है। स्वर के बिना शब्द और शब्द के बिना स्वर अपूर्ण है। दोनों का अन्तर्सम्बन्ध ही उन्हें पूर्ण रूप प्रदान करता है। नाट्यशास्त्र में भरतमुनि ने संगीत की सार्थकता गीत की प्रधानता में बतलाया है। गीत, वाद्य, नृत्य इन तीनों में गीत ही अग्रगामी है शेष अनुगामी।

भरतमुनि कृत "नाट्यशास्त्र में संगीत गायन, वादन, और नर्तन तीनों का संयोग है। रस निष्पत्ति की चर्चा और अध्ययन के लिए संस्कृत और हिंदी ज्ञाताओं के अलावा कला साधकों का इन साइक्लोपीडिया नाट्यशास्त्र ही है। इसमें साहित्य और संगीत के एकत्व को समझा जा सकता है। संगीत और साहित्य, पर्यावरण, चिकित्सा, मनुष्य और समाज से जुड़ा महत्वपूर्ण अनुशासन है।

वैदिक काल से ही काव्य और संगीत में कोई विभेद नहीं था। यज्ञ के अवसर पर वैदिक ऋचाओं के सस्वर पाठ की प्रथा प्रचलित हुई, इन गीत रूप मंत्रों को "साम" कहा गया। प्रतिक्षण परिवर्तनशील जगत की मनुष्य के हृदय पर बड़ी तीव्र प्रतिक्रिया यह होती है की वह 'शाश्वत' और चिरंतन के चक्कर में पड़ जाता है। मनोवैज्ञानिकों के अनुसार वह ऐसे तथ्यों, ऐसे संबंधों की प्यास जगा लेता है जो शाश्वत और सनातन हो। इस प्रतिक्रिया के परिणामस्वरूप उसके मन में अनेकों धारणायें वद्धमूल हो जाती है और उन धारणाओं को वह विवेकपूर्ण समीक्षा करने लग जाता है। काव्य और संगीत में कोई अन्योन्याश्रित सम्बन्ध है, ऐसा विश्वास हमारे मन में अनजाने ही गहरी पैठ बना बैठा है। वैसे तो कला के सभी प्रकार मूल रूप से एक है। उसकी जड़ में एक सहज प्रयोजनातीत आनंदिनी वृत्ति, सृजनात्मकता के लिए एक उत्कृष्ट लालसा, भावाभिव्यक्ति और प्रकाश की दुर्दम अभिलाषा विद्यमान है।

नादेनव्यज्यतेवर्णों पदंवर्णोत्पादाद्वचः।

वचसो व्यवहारोयं नादाधीनमतो जगत।।

संगीत एक त्रिपुटी है।गीत, वाद्य, नृत्य इस त्रयी का समावेश नाद ही के विस्तार है किन्तु इसी के साथ साहित्य भी नाद का विस्तार है। संगीत और साहित्य दोनों ही ललित कलाओं का मूल नाद में है, इसका विवरण प्राचीन ग्रंथों में पाया जाता है। मतंग मुनि ने नाद का विवरण करते हुए 'ततः सर्वचवान्मयम' कह कर इसी बात को स्पष्ट कर दिया।

काव्य और संगीत दोनों के ही मूल में ध्वनि व नाद है। लयात्मक ध्वनि के आधार पर ही संगीत चलता है और ध्वनि से ही शब्द बनते है, जो काव्य के उपजीव्य है तथा छन्द की लयात्मकता के भीतर से होकर गुजरते हैं। संगीत की आधारभूत ध्वनियां सप्त स्वर हैं जो मात्र कम्पनों पर आश्रित हैं। इनका सामाजिक जीवन की वास्तविकताओं और प्रक्रिया से कोई संपर्क नहीं होता। काव्य-साहित्य में ध्वनि-समूहों द्वारा निर्मित शब्द प्रयुक्त होते हैं तथा शब्द अर्थ प्रकाशक होते हैं। उनका सम्बन्ध वाह्य जगत से होता है। शब्द और अर्थ पर विचार करने वाले जानते है कि यह सामजिक संबंधों के प्रतीक हैं। इस सम्बन्ध में 'पं० हजारी प्रसाद जी' का यह कथन दृष्टव्य है, 
-वस्तुतः अर्थहीन छन्द-प्रवाह संगीत ही है।काव्य द्वारा और संगीत द्वारा स्पंदित मानव-चित्त के आवेग में थोड़ा अंतर होता है। काव्य के आवेग द्वारा जो स्पंदन उत्पन्न होता है वह वाह्यसत्ता से पूर्णतया सम्बद्ध होता है।संगीत से उत्पन्न कम्पनों का योग वाह्यसत्ता से कम होने के कारण श्रोताओं के चित्त में उतनी अनुभूति नहीं होती जितनी काव्य जनित आवेगों के कम्पन से होती है।

संगीत ने एक विश्व जनीनता होती है जिसका काव्य में अभाव है। अक्सर जिन भाषाओं व बोलियों का हम एक भी अक्षर नहीं समझ पाते यह वहा के गीतों की 'धुन' ही हम सुन लेते हैं तो आत्मविभोर हो उठते हैं। संगीत की स्वर लहरियां मानव मात्र ही नहीं पशु-पक्षी तक को सम्मोहित कर लेती है, पर काव्य के साथ यह बात नहीं है, वह भाषा की सीमाओं से बंधा है।

उदाहरणार्थ-'निशदिन बरसत नैन हमारे' इतने ही बोल हमें संगीत में पूरा आनन्द दे जाते हैं, परन्तु काव्य का आनन्द लेने के लिए हमें पूरे गीत को पढ़ना ही पड़ेगा। फिर भी संगीत और काव्य साहित्य का घनिष्ठ सम्बन्ध है। किसी एक के बिना दूसरे की रंजकता और रोचकता नष्ट हो जाती है। संगीत यद्यपि एक स्वतंत्र कला है, तथापित जब वह किसी साहित्य से संपृक्त होता है तो उसका उत्कर्ष और भी बढ़ जाता है। संगीत और काव्य साहित्य का उद्देश्य ही है। वैयक्तिक, सामाजिक, राजनीतिक और धार्मिक उत्थान करना।

संगीत वह ललित कला जिसमें एक संगीतज्ञ अपने मनोगत भावों को स्वर, लय, ताल के माध्यम से व्यक्त करता है तथा साहित्य कला में मनोगत भावों को सुन्दर शब्दों के संयोजन से व काव्य रचनाओं के माध्यम से भावों को व्यक्त किया जाता है। यदि अलग-अलग गेयपदों की रचना साहित्य में न की गयी होती तो संगीत में भिन्न-भिन्नगीत शैलियाँ देखने को न मिलती। इन गेय पदों की अपनी निजी विशेषता होती है।

उदाहराणर्थ- ध्रुपद में वीर और रौद्र रस की अधिकता होती है। इन ध्रुपद बंदिशों की रचना भी गंभीर स्वर वाले रागों को ध्यान रखते हुए किया जाता है। साहित्य हमें वीर और रौद्र रस की बंदिशे प्रदान करता है तथा संगीत वीर रस को उद्दीपप्त करता है। जब स्वरों से उत्पन्न भाव अथवा रस, काव्य के द्वारा उत्पन्न भाव एवं रस से मिलता है। तो संगीतज्ञ और श्रोतादानों का हृदय रसाप्लावित हो जाता है। तभी राग मुखरित होकर अपनी रंजकता और रोचकता को सार्थक बनाने में सफल हो पाते है।

ध्रुपद और धमार के अतिरिक्त ख्याल, टप्पा और ठुमरी जैसी गीत शैलियों के प्रकार का निर्वहन तभी हो सकता है जब उनके अनुकूल काव्य रचनाएँ या बंदिशें बनी हो। ख्याल, ठप्पा और ठुमरी जैसी शैलियों में श्रृंगार एवं करूण रस की बहुलता रहती है, अतः इनकी रचना के लिए श्रृंगार और करूण रसानुकूल काव्य साहित्य की आवश्यकता पड़ती है। यदि राग का रसभिन्न है और गेय पदों 
में वर्णित रसभिन्न हो तो ऐसी दशा में रसोत्पत्ति संभव नहीं हो पाती अपितु विरूद्ध रस के प्रयोग से राग की रंजकता और रोचकता नष्ट हो जाती है।

पाश्चात्य विद्वान कार्लाइन के अनुसार-संगीतमय विचार ही काव्य है, कविता मनोवेगपूर्ण और संगीतमय भाषा में मानव भाषा के मानव अन्तःकरण की मूर्त तथा कलात्मक व्यंजना करती है।

अल्फ्रेड आंस्टिन के अनुसार- संगीत से रहित तथाअर्थ की रमणीयता से विहीन शब्दाडम्बर को कविता नहीं कहा जा सकता।

वास्तव में माधुर्य का आविर्भाव विशिष्ट संयोजन में ही निहित होता है। जिस प्रकार एक निश्चित आन्दोलन संख्या के गठन से मधुर स्वर की उत्पत्ति होती है उसी प्रकार नियमित घ्वनि कम्पनों तथा वर्णो के आधार पर मधुर शब्दार्थ की उत्पत्ति होती है। रसालंकार काव्य की स्वर, ताल एवं लय में निबंध रचना ही सुमधुर संगीत है। ऐसा संगीत हृदय की कोमलतम भावनाओं एवं स्पंदन को स्वरों में व्यक्त कर अपने प्रवाह में मानव की आत्मिक भावनाओं को भौतिक लोक से परे अलौकिक लोक में ले जाने की क्षमता रखता है।

"सत्यम् शिवम् सुन्दरम्" का साक्षात्कार कराने वाली इन्हीं कलाओं का नामकरण ललित कला के नाम से हुआ। ललित कलाओं में साहित्य और संगीत का स्थान सर्वश्रेष्ठ रहा। मानव की सहज स्वयंस्फूर्ति प्रेरणाओं को यथार्थ रूप से व्यक्त करने की क्षमता इन्हीं में थी। मानव की भावनाओं का यथार्थ व्यक्तिकरण शब्द व स्वर इन्हीं माध्यमों से हो सका और यही है उनकी सर्वश्रेष्ठता का प्रमाण।

संगीत और साहित्य का अंतर्सबंध पुरातन काल से ही अनवरत चला आ रहा है।दोनों ही कलाओं की आरध्य देवी सरस्वती को हम एक साथ ही वीणावादिनी के रू में पाते हैं। दोनों ही ललित कलाओं का सम्बन्ध विच्छेद एक असम्भव सी बात है। आदिमवेद ऋग्वेद का उदात्त, अनुदात्त और स्वरित आदि स्वरों से अंकित पाया जाना संगीत-साहित्य के गठबंधन के बात को पुष्ट करता हैं। जो भारतीय संस्कृति के प्रतिनिधि विद्वान, साहित्यकार, संगीत के प्रकाण्ड विद्वान रहे उनका यह विश्वास रहा है कि आदिम संगीतज्ञ नटराज की ताल-लय समन्वित ताण्डव से साहित्य व संगीत के तत्वों का एक साथ ही सूत्रपात हुआ। संगीत का आदिराग भैरव भगवान शिव के अघोर मुख से उत्पन्न हुआ और साहित्य की आदि वर्णमाला महादेव के पदचाप से उद्यूत हुई इस प्रकार की परंपरा प्राचीन ग्रंथों में वर्णित है।

जब साहित्य और संगीत के सम्बन्ध की बात होती है तो आशय संगीत की एक विधा से है वह है गायन विधा व कंठ संगीत। वाद्य संगीत तो स्वरों पर आश्रित है, नृत्य अभिनय पर। परगायन का सम्बन्ध साहित्य से अत्यधिक घनिष्ठ है। तराना जैसे अर्थहीन गीतों का गायन नहीं वरन् वर्णमूलक वांगमय की सस्वर अभिव्यक्ति है। संगत और साहित्य के उपादानों की समानता उनके बीच के 
संबंधों पर प्रकाश डालती है। छंदों की झंकृत वेशभूषा, वर्णो तथा अलंकारों का सौष्ठव, लय की रमणीय गति तथा सौन्दर्य बोध दोनों के उपादान कहे जा सकते हैं। संगीत नाद प्रधान है, इसका अर्थ यह है कि उसमें नाद का स्थान प्रथम तथा शब्दों का गोण है, किन्तु शब्दों अथवा भावों के अभाव तथा नगण्यता का वह किसी प्रकार द्योतक नहीं। संगीत नाद प्रधान साहित्य है और साहित्य शब्द प्रधान संगीत।

संस्कृत साहित्य में शब्द-संगीत से झंकृत गीतों की दीर्घ परंपरा रही है। भाव-कल्पना,रस-माधुरी की दृष्टि से संस्कृत का गीतिकाव्य भारत ही नहीं वरन् विश्व की परम श्रेष्ठनिधि है। जयदेवकृत गीतगोविन्द की पदावलियाँ संगीत के विविध रागरागनियों से निबंध है और अभी तक परम आदर के साथ गायी जाती है। गीतिकाव्य की परंपरा जयदेव आदि कवियों से निःसृत होकर हिंदी के विद्यापति, सूर, मीरा, रसखान, तुलसीदास मराठी के समर्थ रामदास, तुकाराम, बंगाल के चैतन्य महाप्रभु, तमिल के त्यागराज आदि की ललित एवं सरस वाणी में शुद्धतम् होकर संगीतज्ञों के लिए गीतों का भण्डारण रूप में प्रस्तुत करती आ रही है।

\section{निष्कर्ष}

संगीत मानव की सहज भावनाओं से उद्वेलन की चेष्टा है। संगीत का नाद साहित्य की चाशनी में घुलकर सम्पूर्ण जगत को रससिक्त करता है। महाकवि कालिदास के 'पवनाग्निसमागम' अर्थात् वायु और अग्नि के संयोग के समान सामर्थ्य संगीत व साहित्य के स्थान को ध्यान में रख कर संगीत से उसका सम्बन्ध अविच्छन्न बना रहे। संगीत व काव्य साहित्य ने समाज को सदैव ही नवजीवन व सार्थक दिशा प्रदान की है। दोनों ही ललित कलाओं के विकास क्रम में वैदिककाल की पवित्रता, रामायण और महाभारत काल की सात्विकता, हिन्दू राजाओं की स्थिरता, मुगलों की ऐय्याशी और कला-परस्त संतों की भक्ति, राजे-रजवाडों की रंगीनियत, अंग्रेजों की उपेक्षा और स्वतंत्र युग के संघर्ष एवं चेतना का प्रभाव हमें दिखलाई पड़ता है। कलाकारों व साहित्यकारों ने युगों-युगों में अपने व्यक्तिगत चिंतन व मनन से जो कुछ पाया अपनीकला में पिरोकर समाज के सामने रखा।

वर्तमान समय में इलेक्ट्रॉनिक मीडिया से जुड़े विशुद्ध समाचार चैनलों में खबरों को शक्तिशाली ढंग से प्रस्तुत करने के लिए प्रत्यक्ष और पार्श्व में संगीत से ही सजाया जाता है। विज्ञापन और फिल्मों की बात ही क्या की जाए? संगीत और साहित्य के सही संगत के बिना इसकी कल्पना भी नहीं की जा सकती। पुराण और इतिहास के दस्तावेजों में दोनों की अहम् भूमिका है। महाकवि सूर्यकांत त्रिपाठी "निराला" के छायावादी गीतों में संगीत के ताल, वाद्य, और नृत्य की उपस्थिति उनके संगीत ज्ञान की परिचायक है। हिंदी साहित्य के उद्भव से आजतक चारण गीतों, सिद्धों के चर्या गीतों, विद्यापति की पदावली और मध्यकालीन सगुण-निर्गुण भक्तों की साहित्य साधना में राग-रागिनियों का चयन व पकड़ मौजूद है। इसके साथ ही अवधि, बुन्देली, पूर्वी, मैथिली आदि बोलियों में रचे लोकगीतों ने भी हिंदी गीतों की पृष्ठभूामि निर्मित की है। मध्यकाल का सम्पूर्ण 
काव्य इसी लोक साहित्य से अनुग्रहित हुआ है। इस प्रकार साहित्य व संगीत एक दूसरे के परस्पर पूरक हैं और भारतीय संगीत में साहित्य का अत्यन्त श्रेष्ठ संयोजन एवं परिमार्जन दृष्टिगोचर होता है।

सन्दर्भ ग्रन्थ सूची

गर्ग, लक्ष्मी नारायण (1989). निबंध संगीत सूर की भक्ति और संगीत, संगीत कार्यालय हाथरस, पृष्ठ संख्या-584 ।

शुक्ल, राम चंद्र. (2016). हिंदी साहित्य का इतिहास, ज्ञान विज्ञान एजुयर,दिल्ली, पृ. संख्या 175। अवस्थी, देवी शंकर (2009). आलोचना और आलोचना, वानी प्रकाशन, दिल्ली, पृ. संख्या 68-73। 